\title{
Delay Induced Hopf Bifurcation of an Epidemic Model with Graded Infection Rates for Internet Worms
}

\author{
Tao Zhao and Dianjie Bi \\ School of Management Science and Engineering, Anhui University of Finance and Economics, \\ Bengbu 233030, China \\ Correspondence should be addressed to Tao Zhao; zhaotaoaufe@163.com
}

Received 1 September 2017; Revised 25 November 2017; Accepted 3 December 2017; Published 24 December 2017

Academic Editor: Marco Spadini

Copyright (C) 2017 Tao Zhao and Dianjie Bi. This is an open access article distributed under the Creative Commons Attribution License, which permits unrestricted use, distribution, and reproduction in any medium, provided the original work is properly cited.

A delayed SEIQRS worm propagation model with different infection rates for the exposed computers and the infectious computers is investigated in this paper. The results are given in terms of the local stability and Hopf bifurcation. Sufficient conditions for the local stability and the existence of Hopf bifurcation are obtained by using eigenvalue method and choosing the delay as the bifurcation parameter. In particular, the direction and the stability of the Hopf bifurcation are investigated by means of the normal form theory and center manifold theorem. Finally, a numerical example is also presented to support the obtained theoretical results.

\section{Introduction}

In the wake of developments in computer technology and communication technology, there is a rapid increase in computer viruses which has brought about huge financial losses [1-3]. Therefore, it is extremely urgent to analyze and protect computers against viruses. In order to understand the spread law of computer viruses over the Internet and in view of the high similarity between computer viruses and biological viruses, many computer virus propagation models have been developed and analyzed. For example, see [4-13] and the cited references therein.

All the models above assume that the infected computer has no infectivity. This is inconsistent with the fact that an infected computer which is in latency can also infect other computers through file copying or file downloading. Based on this, Yang et al. proposed some models [14-17], by taking into account the fact that a computer immediately possesses infectivity once it is infected. However, these models make an assumption that the exposed computers and the infectious computers have the same infectivity. This is not consistent with the reality, because the infection rate of the exposed computers is less than that of the infectious ones. Thus,
Wang et al. [18] proposed the following SEIQRS model with graded infection rates for Internet worms:

$$
\begin{aligned}
\frac{d S(t)}{d t}= & \prod+\gamma R(t)+\theta Q(t)-\beta_{1} S(t) E(t) \\
& -\beta_{2} S(t) I(t)-d S(t), \\
\frac{d E(t)}{d t}= & \beta_{1} S(t) E(t)+\beta_{2} S(t) I(t) \\
& -\left(d+\alpha_{1}+\delta_{2}+\omega\right) E(t), \\
\frac{d I(t)}{d t}= & \omega E(t)-\left(d+\alpha_{2}+(1-p) \delta_{1}+p\right) I(t), \\
\frac{d Q(t)}{d t}= & p I(t)-(d+\eta+\theta) Q(t), \\
\frac{d R(t)}{d t}= & \delta_{2} E(t)+(1-p) \delta_{1} I(t)+\eta Q(t) \\
& -(d+\gamma) R(t),
\end{aligned}
$$

where $S(t), E(t), I(t), Q(t)$, and $R(t)$ denote the numbers of the susceptible, the exposed, the infectious, the quarantined, 
and the recovered computers at time $t$ in the Internet, respectively. $\prod$ is the recruitment of the susceptible computers; $\beta_{1}$ and $\beta_{2}$ are the contact rates of the exposed computers and the infectious computers, respectively; $d$ is the natural death rate of the computers; $\alpha_{1}$ and $\alpha_{2}$ are the death rates of the exposed computers and the infectious computers due to the attack of worms, respectively; $p$ is the quarantined rate of the infectious computers; $\delta_{1}^{-1}$ is the average cured time; $\gamma$, $\theta, \delta_{2}, \omega$, and $\eta$ are the state transition rates. Wang et al. [18] investigated local and global stability of system (1).

It should be pointed out that Wang et al. [18] neglect the fact that it needs a period to clean the worms in the exposed, the infectious, and the quarantined computers for the antivirus software. Time delays cause a stable equilibrium to become unstable and cause Hopf bifurcation phenomenon for a dynamical system. The occurrence of Hopf bifurcation means that the state of worm prevalence changes from an equilibrium point to a limit cycle, which is not welcomed in networks. Therefore, it is meaningful to investigate the effect of time delays on stability of dynamical systems.

As far as we know, there have been some researches on Hopf bifurcation of delayed computer virus models. For example, Feng et al. [19] studied the Hopf bifurcation of a delayed SIRS viral infection model in computer networks by taking the time delay due to the latent and temporary immune period as the bifurcation parameter. Dong et al. [9] proposed a delayed SEIR computer virus model with multistate antivirus and studied the Hopf bifurcation of the model by choosing the delay where the infectious nodes use antivirus software to clean the viruses as the bifurcation parameter. Zhang and Bi [20] investigated the Hopf bifurcation of a delayed computer virus propagation model with infectivity in latent period. For some other research works on the Hopf bifurcation of computer virus models one can refer to [21-24]. Specially, Zhang et al. [24] studied the existence and properties of the Hopf bifurcation of a computer virus model with antidote in vulnerable system by regarding the time delay due to the period that the infected computers use to reinstall system as a bifurcation parameter. Motivated by the work above and considering the effect of the time delay on system (1), we consider the following delayed worm propagation model:

$$
\begin{aligned}
\frac{d S(t)}{d t}= & \prod+\gamma R(t)+\theta Q(t)-\beta_{1} S(t) E(t) \\
& -\beta_{2} S(t) I(t)-d S(t), \\
\frac{d E(t)}{d t}= & \beta_{1} S(t) E(t)+\beta_{2} S(t) I(t) \\
& -\left(d+\alpha_{1}+\omega\right) E(t)-\delta_{2} E(t-\tau), \\
\frac{d I(t)}{d t}= & \omega E(t)-\left(d+\alpha_{2}+p\right) I(t) \\
& -(1-p) \delta_{1} I(t-\tau), \\
\frac{d Q(t)}{d t}= & p I(t)-(d+\theta) Q(t)-\eta Q(t-\tau), \\
\frac{d R(t)}{d t}= & \delta_{2} E(t-\tau)+(1-p) \delta_{1} I(t-\tau)+\eta Q(t-\tau) \\
& -(d+\gamma) R(t),
\end{aligned}
$$

where $\tau$ is the time delay due to the period that the antivirus software uses to clean the worms in the exposed, the infectious, and the quarantined computers.

The object of this paper is to study the existence and properties of the Hopf bifurcation of system (2). The remainder of this paper is organized as follows. The existence of a Hopf bifurcation is discussed by choosing the delay as the bifurcation parameter in Section 2. Properties of the Hopf bifurcation are studied by means of the normal form theory and the center manifold theorem. An example together with its numerical simulations is also presented in order to illustrate the effectiveness of our obtained theoretical results.

\section{Stability of the Viral Equilibrium and Existence of Hopf Bifurcation}

By a simple computation, we know that if $B_{3} \gamma+B_{2} \theta>$ $B_{1}\left(d+\alpha_{1}+\omega+\delta_{2}\right)$ and $d\left(d+\alpha_{1}+\omega+\delta_{2}\right) B_{1}>\prod\left(B_{1} \beta_{1}+\right.$ $\left.\beta_{2}\right)$, then system (2) has a unique endemic equilibrium $D_{*}\left(S_{*}, E_{*}, I_{*}, Q_{*}, R_{*}\right)$, where $S_{*}=\left(A+B_{4} I_{*}\right) / d, E_{*}=B_{1} I_{*}$, $Q_{*}=B_{2} I_{*}, R_{*}=B_{3} I_{*}$, and

$$
\begin{aligned}
I_{*}= & \frac{d\left(d+\alpha_{1}+\omega+\delta_{2}\right) B_{1}-\prod\left(B_{1} \beta_{1}+\beta_{2}\right)}{\beta_{4}\left(B_{1} \beta_{1}+\beta_{2}\right)}, \\
B_{1}= & \frac{d+\alpha_{2}+(1-p) \delta_{1}+p}{\omega}, \\
B_{2}= & \frac{p}{d+\eta+\theta}, \\
B_{3}= & \frac{\delta_{2}\left(d+\alpha_{2}+(1-p) \delta_{1}+p\right)}{\omega(d+\gamma)}+\frac{(1-p) \delta_{1}}{d+\gamma} \\
& +\frac{p \eta}{(d+\gamma)(d+\eta+\theta)}, \\
B_{4}= & B_{3} \gamma+B_{2} \theta-B_{1}\left(d+\alpha_{1}+\omega+\delta_{2}\right) .
\end{aligned}
$$

Then, we obtain the characteristic equation of the linearized system at $D_{*}$ :

$$
\begin{aligned}
\lambda^{5}+ & p_{4} \lambda^{4}+p_{3} \lambda^{3}+p_{2} \lambda^{2}+p_{1} \lambda+p_{0} \\
& +\left(q_{4} \lambda^{4}+q_{3} \lambda^{3}+q_{2} \lambda^{2}+q_{1} \lambda+q_{0}\right) e^{-\lambda \tau} \\
& +\left(r_{3} \lambda^{3}+r_{2} \lambda^{2}+r_{1} \lambda+r_{0}\right) e^{-2 \lambda \tau} \\
& +\left(s_{2} \lambda^{2}+s_{1} \lambda+s_{0}\right) e^{-3 \lambda \tau}=0
\end{aligned}
$$

where

$$
\begin{aligned}
p_{0}= & a_{10} a_{12} a_{13}\left(a_{2} a_{6}-a_{1} a_{7}\right)+a_{9} a_{12} a_{13}\left(a_{1} a_{8}-a_{3} a_{6}\right) \\
& +a_{4} a_{6} a_{9} a_{11} a_{13}, \\
p_{1}= & a_{1} a_{7} a_{10} a_{12}-a_{4} a_{6} a_{9} a_{11} \\
& -a_{8} a_{9}\left(a_{1} a_{12}+a_{1} a_{13}+a_{12} a_{13}\right) \\
& +a_{3} a_{6} a_{9}\left(a_{12}+a_{13}\right)
\end{aligned}
$$




$$
\begin{aligned}
& +a_{13}\left(a_{1} a_{7}\left(a_{10}+a_{12}\right)+a_{10} a_{12}\left(a_{1}+a_{7}\right)\right) \\
& -a_{2} a_{6}\left(a_{10} a_{12}+a_{10} a_{13}+a_{12} a_{13}\right) \text {, } \\
& p_{2}=a_{2} a_{6}\left(a_{10}+a_{12}+a_{13}\right)-a_{1} a_{7}\left(a_{10}+a_{12}\right)-a_{3} a_{6} a_{9} \\
& +a_{8} a_{9}\left(a_{1}+a_{12}+a_{13}\right)-a_{10} a_{12}\left(a_{1}+a_{7}\right) \\
& -a_{13}\left(a_{1} a_{7}+a_{10} a_{12}+\left(a_{1}+a_{7}\right)\left(a_{10}+a_{12}\right)\right) \text {, } \\
& p_{3}=a_{1} a_{7}+a_{10} a_{12}+\left(a_{1}+a_{7}\right)\left(a_{10}+a_{12}\right)-a_{2} a_{6} \\
& -a_{8} a_{9}+a_{13}\left(a_{1}+a_{7}+a_{10}+a_{12}\right) \text {, } \\
& p_{4}=-\left(a_{1}+a_{7}+a_{10}+a_{12}\right) \text {, } \\
& q_{0}=a_{5} a_{6} a_{9}\left(a_{12} b_{5}+a_{11} b_{6}\right)-a_{5} a_{6} a_{10} a_{12} b_{4} \\
& +a_{9} a_{13} b_{3}\left(a_{1} a_{8}-a_{3} a_{6}\right) \\
& +a_{1} a_{13}\left(b_{3}\left(a_{7}+a_{10}\right)+a_{12}\left(b_{1}+b_{2}\right)\right) \\
& -a_{1} a_{7} a_{10} a_{13} b_{3}+a_{2} a_{6} a_{13}\left(a_{10} b_{3}+a_{12} b_{2}\right) \text {, } \\
& q_{1}=a_{7} a_{10} b_{3}\left(a_{1}+a_{13}\right)+a_{5} a_{6} b_{4}\left(a_{10}+a_{12}\right)-a_{5} a_{6} a_{9} b_{5} \\
& -a_{8} a_{9} b_{3}\left(a_{1}+a_{13}\right) \\
& -a_{2} a_{6}\left(a_{13}\left(b_{2}+b_{3}\right)+a_{10} b_{3}+a_{12} b_{2}\right) \text {, } \\
& q_{2}=a_{2} a_{6}\left(b_{2}+b_{3}\right)+a_{8} a_{9} b_{3}-a_{7} a_{10} b_{3} \\
& -a_{1} a_{13}\left(b_{1}+b_{2}+b_{3}\right) \\
& -\left(a_{1}+a_{13}\right)\left(b_{3}\left(a_{7}+a_{10}\right)+a_{12}\left(b_{1}+b_{2}\right)\right) \\
& -a_{5} a_{6} b_{4} \\
& q_{3}=\left(a_{1}+a_{13}\right)\left(b_{1}+b_{2}+b_{3}\right)+b_{3}\left(a_{7}+a_{10}\right) \\
& +a_{12}\left(b_{1}+b_{2}\right) \\
& q_{4}=-\left(b_{1}+b_{2}+b_{3}\right) \text {, } \\
& r_{0}=a_{6} b_{3}\left(a_{5} a_{9} b_{5}+a_{2} a_{13} b_{2}\right)-a_{5} a_{6} b_{4}\left(a_{10} b_{3}+a_{12} b_{2}\right) \\
& -a_{1} a_{13}\left(a_{7} b_{2} b_{3}+a_{10} b_{1} b_{3}+a_{12} b_{1} b_{2}\right) \text {, } \\
& r_{1}=a_{5} a_{6} b_{4}\left(b_{2}+b_{3}\right)-a_{2} a_{6} b_{2} b_{3} \\
& +a_{1} a_{13}\left(b_{1} b_{2}+b_{1} b_{3}+b_{2} b_{3}\right) \\
& +\left(a_{1}+a_{13}\right)\left(a_{7} b_{2} b_{3}+a_{10} b_{1} b_{3}+a_{12} b_{1} b_{2}\right), \\
& r_{2}=-\left(a_{7} b_{2} b_{3}+a_{10} b_{1} b_{3}+a_{12} b_{1} b_{2}\right) \\
& -\left(a_{1}+a_{13}\right)\left(b_{1} b_{2}+b_{1} b_{3}+b_{2} b_{3}\right) \text {, } \\
& r_{3}=b_{1} b_{2}+b_{1} b_{3}+b_{2} b_{3} \text {, } \\
& s_{0}=-b_{2} b_{3}\left(a_{5} a_{6} b_{4}+a_{1} a_{13} b_{1}\right) \text {, } \\
& s_{1}=b_{1} b_{2} b_{3}\left(a_{1}+a_{13}\right) \text {, } \\
& s_{2}=-b_{1} b_{2} b_{3} \text {, }
\end{aligned}
$$

$$
\begin{aligned}
& a_{1}=-\left(\beta_{1} E_{*}+\beta_{2} I_{*}+d\right), \\
& a_{2}=-\beta_{1} S_{*}, \\
& a_{3}=-\beta_{2} S_{*}, \\
& a_{4}=\theta \\
& a_{5}=\gamma \\
& a_{6}=\beta_{1} E_{*}+\beta_{2} I_{*}, \\
& a_{7}=\beta_{1} S_{*}-\left(d+\alpha_{1}+\omega\right), \\
& a_{8}=\beta_{2} S_{*}, \\
& a_{9}=\omega \\
& a_{10}=-\left(d+\alpha_{2}+p\right), \\
& a_{11}=p \\
& a_{12}=-(d+\theta) \\
& a_{13}=-(d+\gamma) \\
& b_{1}=-\delta_{2} \\
& b_{2}=-(1-p) \delta_{1}, \\
& b_{3}=-\eta \\
& b_{4}=\delta_{2}, \\
& b_{5}=(1-p) \delta_{1}, \\
& b_{6}=\eta \\
& =
\end{aligned}
$$

For $\tau=0$, (4) becomes

$$
\lambda^{5}+A_{4} \lambda^{4}+A_{3} \lambda^{3}+A_{2} \lambda^{2}+A_{1} \lambda+A_{0}=0
$$

with

$$
\begin{aligned}
& A_{0}=p_{0}+q_{0}+r_{0}+s_{0}, \\
& A_{1}=p_{1}+q_{1}+r_{1}+s_{1}, \\
& A_{2}=p_{2}+q_{2}+r_{2}+s_{2}, \\
& A_{3}=p_{3}+q_{3}+r_{3}, \\
& A_{4}=p_{4}+q_{4} .
\end{aligned}
$$

According to Routh-Hurwitz criteria when the condition $\left(H_{1}\right)$ is satisfied, that is, when (8)-(12) hold, then $D_{*}\left(S_{*}, E_{*}, I_{*}, Q_{*}, R_{*}\right)$ is locally asymptotically stable when $\tau=0$.

$$
\begin{aligned}
& \operatorname{det}_{1}=A_{4}>0, \\
& \operatorname{det}_{2}=\left(\begin{array}{ll}
A_{4} & 1 \\
A_{2} & A_{3}
\end{array}\right)>0,
\end{aligned}
$$




$$
\begin{aligned}
\operatorname{det}_{3} & =\left(\begin{array}{ccc}
A_{4} & 1 & 0 \\
A_{2} & A_{3} & A_{4} \\
0 & A_{1} & A_{2}
\end{array}\right)>0, \\
\operatorname{det}_{4} & =\left(\begin{array}{cccc}
A_{4} & 1 & 0 & 0 \\
A_{2} & A_{3} & A_{4} & 1 \\
A_{0} & A_{1} & A_{2} & A_{3} \\
0 & 0 & A_{0} & A_{1}
\end{array}\right)>0, \\
\operatorname{det}_{5} & =\left(\begin{array}{ccccc}
A_{4} & 1 & 0 & 0 & 0 \\
A_{2} & A_{3} & A_{4} & 1 & 0 \\
A_{0} & A_{1} & A_{2} & A_{3} & A_{4} \\
0 & 0 & A_{0} & A_{1} & A_{2} \\
0 & 0 & 0 & 0 & A_{0}
\end{array}\right)>0 .
\end{aligned}
$$

Multiplying $e^{\lambda \tau}$ on both sides of (4), one can get

$$
\begin{aligned}
q_{4} \lambda^{4} & +q_{3} \lambda^{3}+q_{2} \lambda^{2}+q_{1} \lambda+q_{0} \\
& +\left(\lambda^{5}+p_{4} \lambda^{4}+p_{3} \lambda^{3}+p_{2} \lambda^{2}+p_{1} \lambda+p_{0}\right) e^{\lambda \tau} \\
& +\left(r_{3} \lambda^{3}+r_{2} \lambda^{2}+r_{1} \lambda+r_{0}\right) e^{-\lambda \tau} \\
& +\left(s_{2} \lambda^{2}+s_{1} \lambda+s_{0}\right) e^{-2 \lambda \tau}=0 .
\end{aligned}
$$

For $\tau>0$, we assume that $\lambda=i \omega(\omega>0)$ is the root of (13); then

$$
\begin{aligned}
& s_{1} \omega \sin 2 \tau \omega+\left(s_{0}-s_{2} \omega^{2}\right) \cos 2 \tau \omega=G_{1}(\omega), \\
& s_{1} \omega \cos 2 \tau \omega-\left(s_{0}-s_{2} \omega^{2}\right) \sin 2 \tau \omega=G_{2}(\omega),
\end{aligned}
$$

where

$$
\begin{aligned}
G_{1}(\omega)= & \left(\omega^{5}-\left(p_{3}+r_{3}\right) \omega^{3}+\left(p_{1}-r_{1}\right) \omega\right) \sin \tau \omega \\
& -\left(p_{4} \omega^{4}-\left(p_{2}+r_{2}\right) \omega^{2}+p_{0}+r_{0}\right) \cos \tau \omega \\
& +q_{2} \omega^{2}-q_{4} \omega^{4}-q_{0}, \\
G_{2}(\omega)= & -\left(\omega^{5}-\left(p_{3}+r_{3}\right) \omega^{3}+\left(p_{1}+r_{1}\right) \omega\right) \cos \tau \omega \\
& -\left(p_{4} \omega^{4}-\left(p_{2}-r_{2}\right) \omega^{2}+p_{0}-r_{0}\right) \sin \tau \omega \\
& +q_{3} \omega^{3}-q_{1} \omega .
\end{aligned}
$$

Then,

$$
\begin{gathered}
c_{0}(\omega)+c_{1}(\omega) \sin \tau \omega+c_{2}(\omega) \sin \tau \omega \cos \tau \omega \\
+c_{3}(\omega) \cos \tau \omega+c_{4}(\omega) \cos ^{2} \tau \omega=0,
\end{gathered}
$$

with

$$
\begin{aligned}
& c_{0}(\omega)=\omega^{10}+\left(p_{4}^{2}+q_{4}^{2}-2\left(p_{3}+r_{3}\right)\right) \omega^{8} \\
& +\left(\left(p_{3}+r_{3}\right)^{2}+q_{3}^{2}+2 p_{1}-2 r_{1}-2 p_{2} p_{4}-2 p_{4} r_{2}\right. \\
& \left.-2 q_{2} q_{4}\right) \omega^{6}+\left(p_{2}^{2}+q_{2}^{2}+r_{2}^{2}-s_{2}^{2}+2 p_{0} p_{4}+2 p_{2} r_{2}\right. \\
& \left.+2 p_{4} r_{0}+2 q_{0} q_{4}-2 q_{1} q_{3}-2\left(p_{1}+r_{1}\right)\left(p_{3}+r_{3}\right)\right) \\
& \cdot \omega^{4}+\left(p_{1}^{2}+q_{1}^{2}+r_{1}^{2}-s_{1}^{2}-2 p_{0} p_{2}-2 q_{0} q_{2}-2 r_{0} r_{2}\right. \\
& \left.-2 p_{1} r_{1}\right) \omega^{2}+p_{0}^{2}+q_{0}^{2}+r_{0}^{2}-s_{0}^{2}+2 p_{0} r_{0} \text {, } \\
& c_{1}(\omega)=-2 q_{4} \omega^{9}+2\left(q_{2}-p_{4} q_{3}+q_{4}\left(p_{3}+r_{3}\right)\right) \omega^{7} \\
& +2\left(p_{4} q_{1}-q_{0}+q_{3}\left(p_{2}-r_{2}\right)-q_{2}\left(p_{3}+r_{3}\right)\right. \\
& \left.-n_{4}\left(p_{1}-r_{1}\right)\right) \omega^{5}+2\left(q_{0}\left(p_{3}+r_{3}\right)+q_{2}\left(p_{1}-r_{1}\right)\right. \\
& \left.-q_{1}\left(p_{2}-r_{2}\right)-q_{3}\left(p_{0}-r_{0}\right)\right) \omega^{3}+2\left(q_{1}\left(p_{0}-r_{0}\right)\right. \\
& \left.-n_{0}\left(p_{1}-r_{1}\right)\right) \omega \\
& c_{2}(\omega)=-4 r_{2} \omega^{7}+4\left(r_{0}-p_{4} r_{1}+r_{2}\left(p_{3}+r_{3}\right)\right) \omega^{5} \\
& -4\left(r_{0}\left(p_{3}+r_{3}\right)+r_{1} r_{2}+p_{2} r_{1}\right) \omega^{3}+4\left(p_{1} r_{0}\right. \\
& \left.-p_{0} r_{1}\right) \omega \\
& c_{3}(\omega)=2\left(p_{4} q_{4}-q_{3}\right) \omega^{8}+2\left(q_{1}-p_{4} q_{2}\right. \\
& \left.+q_{3}\left(p_{3}+q_{3}\right)-q_{4}\left(p_{2}+r_{2}\right)\right) \omega^{6}+2\left(p_{4} q_{0}\right. \\
& +q_{2}\left(p_{2}+r_{2}\right)+q_{4}\left(p_{0}+r_{0}\right)-q_{1}\left(p_{3}+r_{3}\right) \\
& \left.-q_{3}\left(p_{1}+r_{1}\right)\right) \omega^{4}+2\left(q_{1}\left(p_{1}+r_{1}\right)-q_{2}\left(p_{0}+r_{0}\right)\right) \\
& \cdot \omega^{2}+2 q_{0}\left(p_{0}+r_{0}\right) \\
& c_{4}(\omega)=4\left(r_{1}+p_{4} r_{2}\right) \omega^{6}+4\left(p_{2} r_{2}+p_{4} r_{0}\right. \\
& \left.-r_{1}\left(p_{3}+r_{3}\right)\right) \omega^{4}+4 p_{1} r_{1} \omega^{2}-4 p_{0} r_{0} .
\end{aligned}
$$

Since $\sin \tau \omega= \pm \sqrt{1-\cos ^{2} \tau \omega},(16)$ can be transformed into $c_{0}(\omega)+c_{3}(\omega) \cos \tau \omega+c_{4}(\omega) \cos ^{2} \tau \omega$

$$
\begin{aligned}
& = \pm\left(c_{1}(\omega) \sqrt{1-\cos ^{2} \tau \omega}\right. \\
& \left.+c_{2}(\omega) \sqrt{1-\cos ^{2} \tau \omega} \cos \tau \omega\right) .
\end{aligned}
$$

It equals

$$
\begin{gathered}
e_{4}(\omega) \cos ^{4} \tau \omega+e_{3}(\omega) \cos ^{3} \tau \omega+e_{2}(\omega) \cos ^{2} \tau \omega \\
+e_{1}(\omega) \cos \tau \omega+e_{0}(\omega)=0,
\end{gathered}
$$

where

$$
\begin{aligned}
& e_{0}(\omega)=c_{0}^{2}(\omega)-c_{1}^{2}(\omega), \\
& e_{1}(\omega)=2\left(c_{0}(\omega) \times c_{3}(\omega)-c_{1}(\omega) \times c_{2}(\omega)\right),
\end{aligned}
$$




$$
\begin{aligned}
& e_{2}(\omega)=c_{3}^{2}(\omega)+2 c_{0}(\omega) \times c_{4}(\omega)+c_{1}^{2}(\omega)-c_{2}^{2}(\omega), \\
& e_{3}(\omega)=2\left(c_{3}(\omega) \times c_{4}(\omega)+c_{1}(\omega) \times c_{2}(\omega)\right), \\
& e_{4}(\omega)=c_{4}^{2}(\omega)+c_{2}^{2}(\omega) .
\end{aligned}
$$

Based on the discussion about the distribution of the root of $(19)$ in $[25,26]$, we obtain the expression of $\cos \tau \omega$, say

$$
\cos \tau \omega=f_{1}(\omega) .
$$

Substitute (21) into (16), then the expression of $\sin \tau \omega$ can be obtained, say

$$
\sin \tau \omega=f_{2}(\omega)
$$

Hence, we have

$$
f_{1}^{2}(\omega)+f_{2}^{2}(\omega)=1
$$

In order to obtain the main results in this paper, we suppose that $\left(\mathrm{H}_{2}\right)((23))$ has at least one positive root $\omega_{0}$. For $\omega_{0}$, we have

$$
\tau_{0}=\frac{1}{\omega_{0}}\left\{\arccos f_{1}\left(\omega_{0}\right)\right\}
$$

Differentiating (13) with respect to $\tau$, we obtain

$$
\left[\frac{d \lambda}{d \tau}\right]^{-1}=\frac{U(\lambda)}{V(\lambda)}-\frac{\tau}{\lambda},
$$

where

$$
\begin{aligned}
U(\lambda) & \\
= & 4 q_{4} \lambda^{3}+3 q_{3} \lambda^{2}+2 q_{2} \lambda+q_{1} \\
& +\left(5 \lambda^{4}+4 p_{4} \lambda^{3}+3 p_{3} \lambda^{2}+2 p_{2} \lambda+p_{1}\right) e^{\lambda \tau} \\
& +\left(3 r_{3} \lambda^{2}+2 r_{2} \lambda+r_{1}\right) e^{-\lambda \tau}+\left(2 s_{2} \lambda+s_{1}\right) e^{-2 \lambda \tau}, \\
V(\lambda) & \\
= & 2\left(s_{2} \lambda^{3}+s_{1} \lambda^{2}+s_{0} \lambda\right) e^{-2 \lambda \tau} \\
& +\left(r_{3} \lambda^{4}+r_{2} \lambda^{3}+r_{1} \lambda^{2}+r_{0} \lambda\right) e^{-\lambda \tau} \\
& -\left(\lambda^{6}+p_{4} \lambda^{5}+p_{3} \lambda^{4}+p_{2} \lambda^{3}+p_{1} \lambda^{2}+p_{0} \lambda\right) e^{\lambda \tau} .
\end{aligned}
$$

Further,

$$
\begin{aligned}
\operatorname{Re}\left[\frac{d \lambda}{d \tau}\right]_{\lambda=i \omega_{0}}^{-1}=\frac{U_{R} \times V_{R}+U_{I} \times V_{I}}{V_{R}^{2}+V_{I}^{2}}, \\
U_{R} \\
=\left(5 \omega_{0}^{4}-3\left(p_{3}-r_{3}\right) \omega_{0}^{2}+p_{1}+r_{1}\right) \cos \tau_{0} \omega_{0} \\
\quad-2\left(\left(p_{2}-r_{2}\right) \omega_{0}-2 p_{4} \omega_{0}^{3}\right) \sin \tau_{0} \omega_{0} \\
\quad+2 s_{2} \omega_{0} \sin 2 \tau_{0} \omega_{0}+s_{1} \cos 2 \tau_{0} \omega_{0}+q_{1}-3 q_{3} \omega_{0}^{2},
\end{aligned}
$$

$$
\begin{aligned}
U_{I} & \\
= & \left(5 \omega_{0}^{4}-3\left(p_{3}-r_{3}\right) \omega_{0}^{2}+p_{1}-r_{1}\right) \sin \tau_{0} \omega_{0} \\
& +2\left(\left(p_{2}+r_{2}\right) \omega_{0}-2 p_{4} \omega_{0}^{3}\right) \cos \tau_{0} \omega_{0} \\
& +2 s_{2} \omega_{0} \cos 2 \tau_{0} \omega_{0}-s_{1} \sin 2 \tau_{0} \omega_{0}+2 q_{2} \omega_{0} \\
& -4 q_{4} \omega_{0}^{3}, \\
V_{R} \quad & \\
= & \left(\omega_{0}^{4}-\left(p_{3}-r_{3}\right) \omega_{0}^{2}+p_{1}-r_{1}\right) \omega_{0}^{2} \cos \tau_{0} \omega_{0} \\
& -\left(\left(p_{2}+r_{2}\right) \omega_{0}^{2}-p_{4} \omega_{0}^{4}-p_{0}-r_{0}\right) \sin \tau_{0} \omega_{0} \\
& +\left(s_{0} \omega_{0}-s_{2} \omega_{0}^{3}\right) \sin 2 \tau_{0} \omega_{0}-2 s_{1} \omega_{0} \cos 2 \tau_{0} \omega_{0},
\end{aligned}
$$

$V_{I}$

$$
\begin{aligned}
= & \left(\omega_{0}^{4}-\left(p_{3}+r_{3}\right) \omega_{0}^{2}+p_{1}+r_{1}\right) \omega_{0}^{2} \sin \tau_{0} \omega_{0} \\
& +\left(\left(p_{2}-r_{2}\right) \omega_{0}^{2}-p_{4} \omega_{0}^{4}-p_{0}+r_{0}\right) \cos \tau_{0} \omega_{0} \\
& +\left(s_{0} \omega_{0}-s_{2} \omega_{0}^{3}\right) \cos 2 \tau_{0} \omega_{0}+2 s_{1} \omega_{0} \sin 2 \tau_{0} \omega_{0} .
\end{aligned}
$$

Therefore, if condition $\left(H_{3}\right), G_{1 R} \times G_{2 R}+G_{1 I} \times G_{2 I} \neq 0$, holds, then $\operatorname{Re}[d \lambda / d \tau]_{\lambda=i \omega_{0}} \neq 0$. Based on the Hopf bifurcation theorem in [27], we have the following.

Theorem 1. For system (2), if conditions $\left(H_{1}\right),\left(H_{2}\right)$, and $\left(H_{3}\right)$ hold, then the endemic equilibrium $D_{*}\left(S_{*}, E_{*}, I_{*}, Q_{*}, R_{*}\right)$ is locally asymptotically stable when $\tau \in\left[0, \tau_{0}\right)$; a Hopf bifurcation occurs at the endemic equilibrium $D_{*}\left(S_{*}, E_{*}, I_{*}, Q_{*}, R_{*}\right)$ when $\tau=\tau_{0}$ and a family of periodic solutions bifurcate from the endemic equilibrium $D_{*}\left(S_{*}, E_{*}, I_{*}, Q_{*}, R_{*}\right)$ near $\tau=\tau_{0}$.

\section{Direction and Stability of the Hopf Bifurcation}

In this section, we describe the direction, stability, and period of the Hopf bifurcation of system (2) from the endemic equilibrium $D_{*}\left(S_{*}, E_{*}, I_{*}, Q_{*}, R_{*}\right)$ based on the normal form theory and center manifold theorem given by Hassard et al. [27]. Let $u_{1}(t)=S(t)-S_{*}, u_{2}(t)=E(t)-E_{*}, u_{3}(t)=I(t)-I_{*}$, $u_{4}(t)=Q(t)-Q_{*}$, and $u_{5}(t)=R(t)-R_{*}$, and normalize the time delay by $t \rightarrow(t / \tau)$. And let $\tau=\tau_{0}+\varrho, \varrho \in R$, so that the value $\varrho=0$ is the Hopf bifurcation value. Define the space of continuous real valued functions as $C=C\left([-1,0], R^{5}\right)$. Then, the delayed system (2) can be transformed into the functional differential equation in $C$ :

$$
\dot{u}(t)=L_{\varrho} u_{t}+F\left(\varrho, u_{t}\right),
$$

where $u_{t}=\left(u_{1}(t), u_{2}(t), u_{3}(t), u_{4}(t), u_{5}(t)\right)^{T}=(S, E, I, Q$, $R)^{T} \in R^{5}, u_{t}(\theta)=u(t+\theta) \in C, L_{\varrho}: C \rightarrow R^{5}$, and $F\left(\varrho, u_{t}\right) \rightarrow$ $R^{5}$ are given, respectively, by

$$
L_{\varrho} \phi=\left(\tau_{0}+\varrho\right)\left(A_{\max } \phi(0)+B_{\max } \phi(-1)\right),
$$


with

$$
\begin{aligned}
& A_{\max }=\left(\begin{array}{ccccc}
a_{1} & a_{2} & a_{3} & a_{4} & a_{5} \\
a_{6} & a_{7} & a_{8} & 0 & 0 \\
0 & a_{9} & a_{10} & 0 & 0 \\
0 & 0 & a_{11} & a_{12} & 0 \\
0 & 0 & 0 & 0 & a_{13}
\end{array}\right) \\
& B_{\max }=\left(\begin{array}{ccccc}
0 & 0 & 0 & 0 & 0 \\
0 & b_{1} & 0 & 0 & 0 \\
0 & 0 & b_{2} & 0 & 0 \\
0 & 0 & 0 & b_{3} & 0 \\
0 & b_{4} & b_{5} & b_{6} & 0
\end{array}\right) \text {, } \\
& F(\varrho, \phi)=\left(\tau_{0}+\varrho\right) \\
& \left(\begin{array}{c}
-\left(\beta_{1} \phi_{1}(0) \phi_{2}(0)+\beta_{2} \phi_{1}(0) \phi_{3}(0)\right) \\
\beta_{1} \phi_{1}(0) \phi_{2}(0)+\beta_{2} \phi_{1}(0) \phi_{3}(0) \\
0 \\
0 \\
0
\end{array}\right) .
\end{aligned}
$$

By the Riesz representation theorem, there exists a function $\eta(\theta, \varrho)$ whose components are of bounded variation for $\theta \in[-1,0]$ such that

$$
L_{\mu} \phi=\int_{-1}^{0} d \eta(\theta, \varrho) \phi(\theta),
$$

for $\phi \in C$. In view of (29), we can choose

$$
\eta(\theta, \varrho)=\left(\tau_{0}+\varrho\right)\left(A_{\max } \delta(\theta)+B_{\max } \delta(\theta+1)\right),
$$

with $\delta(\theta)$ being the Dirac delta function.

For $\phi \in C\left([-1,0], R^{5}\right)$, define

$$
\begin{aligned}
& A(\varrho) \phi= \begin{cases}\frac{d \phi(\theta)}{d \theta}, & -1 \leq \theta<0, \\
\int_{-1}^{0} d \eta(\theta, \varrho) \phi(\theta), & \theta=0,\end{cases} \\
& R(\varrho) \phi= \begin{cases}0, & -1 \leq \theta<0, \\
F(\varrho, \phi), & \theta=0 .\end{cases}
\end{aligned}
$$

Then, system (28) is then equivalent to

$$
\dot{u}(t)=A(\varrho) u_{t}+R(\varrho) u_{t} .
$$

For $\varphi \in C^{1}\left([0,1],\left(R^{5}\right)^{*}\right)$, define

$$
A^{*}(\varphi)= \begin{cases}-\frac{d \varphi(s)}{d s}, & 0<s \leq 1, \\ \int_{-1}^{0} d \eta^{T}(s, 0) \varphi(-s), & s=0,\end{cases}
$$

and a bilinear inner product

$$
\begin{aligned}
\langle\varphi(s), \phi(\theta)\rangle= & \bar{\varphi}(0) \phi(0) \\
& -\int_{\theta=-1}^{0} \int_{\xi=0}^{\theta} \bar{\varphi}(\xi-\theta) d \eta(\theta) \phi(\xi) d \xi,
\end{aligned}
$$

where $\eta(\theta)=\eta(\theta, 0)$. Then $A(0)$ (from here onwards we refer $A(0)$ by $A)$ and $A^{*}$ are adjoint operators. Since $\pm i \omega_{0} \tau_{0}$ are the eigenvalues of $A$, they are also the eigenvalues of $A^{*}$.

Suppose that $\rho(\theta)=\left(1, \rho_{2}, \rho_{3}, \rho_{4}, \rho_{5}\right)^{T} e^{i \tau_{0} \omega_{0} \theta}$ and $\rho^{*}(s)=$ $\left(1, \rho_{2}^{*}, \rho_{3}^{*}, \rho_{4}^{*}, \rho_{5}^{*}\right)^{T} e^{i \tau_{0} \omega_{0} s}$ are the eigenvectors for $A$ and $A^{*}$ corresponding to $+i \tau_{0} \omega_{0}$ and $-i \tau_{0} \omega_{0}$, respectively. Then, we have

$$
\begin{aligned}
& \rho_{2} \\
& =\frac{a_{6}\left(i \omega_{0}-a_{10}-b_{2} e^{-i \tau_{0} \omega_{0}}\right)}{\left(i \omega_{0}-a_{7}-b_{1} e^{-i \tau_{0} \omega_{0}}\right)\left(i \omega_{0}-a_{10}-b_{2} e^{-i \tau_{0} \omega_{0}}\right)-a_{8} a_{9}}, \\
& \rho_{3}=\frac{a_{9} \rho_{2}}{i \omega_{0}-a_{10}-b_{2} e^{-i \tau_{0} \omega_{0}}}, \\
& \rho_{4}=\frac{a_{11} \rho_{3}}{i \omega_{0}-a_{12}-b_{3} e^{-i \tau_{0} \omega_{0}}}, \\
& \rho_{5}=\frac{b_{4} \rho_{2}+b_{5} \rho_{3}+b_{6} \rho_{4}}{\left(i \omega_{0}-a_{13}\right) e^{i \tau_{0} \omega_{0}}}, \\
& \rho_{2}^{*}=-\frac{i \omega_{0}+a_{1}}{a_{6}}, \\
& \rho_{3}^{*}=-\frac{a_{3}+a_{8} \rho_{2}^{*}+a_{11} \rho_{4}^{*}+b_{5} e^{i \tau_{0} \omega_{0}} \rho_{5}^{*}}{i \omega_{0}+a_{10}+b_{2} e^{i \tau_{0} \omega_{0}}}, \\
& \rho_{4}^{*}=-\frac{a_{4}+b_{6} e^{i \tau_{0} \omega_{0}} \rho_{5}^{*}}{i \omega_{0}+a_{12}+b_{3} e^{i \tau_{0} \omega_{0}}}, \\
& \rho_{5}^{*}=-\frac{a_{5}}{i \omega_{0}+a_{13}} .
\end{aligned}
$$

From (37), we get

$$
\begin{aligned}
\bar{V} & =\left[1+\rho_{2} \bar{\rho}_{2}^{*}+\rho_{3} \bar{\rho}_{3}^{*}+\rho_{4} \bar{\rho}_{4}^{*}+\rho_{5} \bar{\rho}_{5}^{*}\right. \\
& +\tau_{0} e^{-i \tau_{0} \omega_{0}}\left(\rho_{2}\left(b_{1} \bar{\rho}_{2}^{*}+b_{4} \bar{\rho}_{5}^{*}\right)+\rho_{3}\left(b_{2} \bar{\rho}_{3}^{*}+b_{5} \bar{\rho}_{5}^{*}\right)\right. \\
& \left.\left.+\rho_{4}\left(b_{3} \bar{\rho}_{4}^{*}+b_{6} \bar{\rho}_{5}^{*}\right)\right)\right]^{-1}
\end{aligned}
$$

such that $\left\langle\rho^{*}, \rho\right\rangle=1$ and $\left\langle\rho^{*}, \bar{\rho}\right\rangle=0$.

Proceeding in the same manner as Hassard et al. [27] and the similar computation process as that in [28-30], we obtain

$$
\begin{aligned}
& g_{20}=2 \tau_{0} \bar{V}\left(\bar{\rho}_{2}^{*}-1\right)\left(\beta_{1} \rho_{2}+\beta_{2} \rho_{3}\right), \\
& g_{11}=\tau_{0} \bar{V}\left(\bar{\rho}_{2}^{*}-1\right)\left(\beta_{1} \operatorname{Re}\left\{\rho_{2}\right\}+\beta_{2} \operatorname{Re}\left\{\rho_{3}\right\}\right), \\
& g_{02}=2 \tau_{0} \bar{V}\left(\bar{\rho}_{2}^{*}-1\right)\left(\beta_{1} \bar{\rho}_{2}+\beta_{2} \bar{\rho}_{3}\right), \\
& g_{21}=2 \tau_{0} \bar{V}\left(\bar{\rho}_{2}^{*}-1\right)\left(\beta _ { 1 } \left(W_{11}^{(1)}(0) \rho_{2}+\frac{1}{2} W_{20}^{(1)}(0) \bar{\rho}_{2}\right.\right.
\end{aligned}
$$




$$
\begin{aligned}
& \left.+W_{11}^{(2)}(0)+\frac{1}{2} W_{20}^{(2)}(0)\right)+\beta_{2}\left(W_{11}^{(1)}(0) \rho_{3}\right. \\
& \left.\left.+\frac{1}{2} W_{20}^{(1)}(0) \bar{\rho}_{3}+W_{11}^{(3)}(0)+\frac{1}{2} W_{20}^{(3)}(0)\right)\right),
\end{aligned}
$$

with

$$
\begin{aligned}
W_{20}(\theta)= & \frac{i g_{20} \rho(0)}{\tau_{0} \omega_{0}} e^{i \tau_{0} \omega_{0} \theta}+\frac{i \bar{g}_{02} \bar{\rho}(0)}{3 \tau_{0} \omega_{0}} e^{-i \tau_{0} \omega_{0} \theta} \\
& +E_{1} e^{2 i \tau_{0} \omega_{0} \theta}, \\
W_{11}(\theta)= & -\frac{i g_{11} \rho(0)}{\tau_{0} \omega_{0}} e^{i \tau_{0} \omega_{0} \theta}+\frac{i \bar{g}_{11} \bar{\rho}(0)}{\tau_{0} \omega_{0}} e^{-i \tau_{0} \omega_{0} \theta}+E_{2} .
\end{aligned}
$$

$E_{1}$ and $E_{2}$ can be obtained by the following two equations:

$E_{1}$

$$
\begin{aligned}
& =2\left(\begin{array}{ccccc}
a_{1}^{\prime} & -a_{2} & -a_{3} & -a_{4} & -a_{5} \\
-a_{6} & a_{7}^{\prime} & -a_{8} & 0 & 0 \\
0 & -a_{9} & a_{10}^{\prime} & 0 & 0 \\
0 & 0 & -a_{11} & a_{12}^{\prime} & 0 \\
0 & -b_{4} e^{-2 i \tau_{0} \omega_{0}} & -b_{5} e^{-2 i \tau_{0} \omega_{0}} & -b_{6} e^{-2 i \tau_{0} \omega_{0}} & a_{13}^{\prime}
\end{array}\right)^{-1} \\
& \times\left(\begin{array}{c}
E_{1}^{(1)} \\
E_{1}^{(2)} \\
0 \\
0 \\
0
\end{array}\right),
\end{aligned}
$$

$E_{2}$

$$
\begin{aligned}
& =\left(\begin{array}{ccccc}
a_{1} & a_{2} & a_{3} & a_{4} & a_{5} \\
a_{6} & a_{7}+b_{1} & a_{8} & 0 & 0 \\
0 & a_{9} & a_{10}+b_{2} & 0 & 0 \\
0 & 0 & a_{11} & a_{12}+b_{3} & 0 \\
0 & b_{4} & b_{5} & b_{6} & 0
\end{array}\right)^{-1} \\
& \times\left(\begin{array}{c}
E_{2}^{(1)} \\
E_{2}^{(2)} \\
0 \\
0 \\
0
\end{array}\right),
\end{aligned}
$$

where

$$
\begin{aligned}
& a_{1}^{\prime}=2 i \omega_{0}-a_{1}, \\
& a_{7}^{\prime}=2 i \omega_{0}-a_{7}-e^{-2 i \tau_{0} \omega_{0},}, \\
& a_{10}^{\prime}=2 i \omega_{0}-a_{10}-b_{2} e^{-2 i \tau_{0} \omega_{0},}, \\
& a_{12}^{\prime}=2 i \omega_{0}-a_{12}-b_{3} e^{-2 i \tau_{0} \omega_{0}}, \\
& a_{13}^{\prime}=2 i \omega_{0}-a_{13},
\end{aligned}
$$

$$
\begin{aligned}
& E_{1}^{(1)}=-\beta_{1} \rho_{2}-\beta_{2} \rho_{3}, \\
& E_{1}^{(2)}=\beta_{1} \rho_{2}+\beta_{2} \rho_{3}, \\
& E_{2}^{(1)}=-\beta_{1} \operatorname{Re}\left\{\rho_{2}\right\}-\beta_{2} \operatorname{Re}\left\{\rho_{3}\right\}, \\
& E_{2}^{(2)}=\beta_{1} \operatorname{Re}\left\{\rho_{2}\right\}+\beta_{2} \operatorname{Re}\left\{\rho_{3}\right\} .
\end{aligned}
$$

Then, one can obtain

$$
\begin{aligned}
C_{1}(0) & =\frac{i}{2 \tau_{0} \omega_{0}}\left(g_{11} g_{20}-2\left|g_{11}\right|^{2}-\frac{\left|g_{02}\right|^{2}}{3}\right)+\frac{g_{21}}{2} \\
\mu_{2} & =-\frac{\operatorname{Re}\left\{C_{1}(0)\right\}}{\operatorname{Re}\left\{\lambda^{\prime}\left(\tau_{0}\right)\right\}} \\
\beta_{2} & =2 \operatorname{Re}\left\{C_{1}(0)\right\} \\
T_{2} & =-\frac{\operatorname{Im}\left\{C_{1}(0)\right\}+\mu_{2} \operatorname{Im}\left\{\lambda^{\prime}\left(\tau_{0}\right)\right\}}{\tau_{0} \omega_{0}} .
\end{aligned}
$$

Thus, based on the properties of the Hopf bifurcation discussed in [27], we have the following.

Theorem 2. For system (2), if $\mu_{2}>0\left(\mu_{2}<0\right)$, then the Hopf bifurcation is supercritical (subcritical); if $\beta_{2}<0\left(\beta_{2}>0\right)$, then the bifurcated periodic solutions are stable (unstable); if $T_{2}>0\left(T_{2}<0\right)$, then the period of the bifurcated periodic solutions increases (decreases).

\section{Numerical Simulation}

In this section, we perform some numerical simulations to support and explain our obtained results. We choose a set of parameters as follows: $\Pi=5, \gamma=0.6, \theta=0.3, \beta_{1}=0.0002$, $\beta_{2}=0.0003, d=0.0001, \alpha_{1}=0.0001, \omega=0.01, \delta_{1}=0.8$, $\delta_{2}=0.2, \alpha_{2}=0.0002, p=0.4$, and $\eta=0.35$. Then, system (2) becomes

$$
\begin{aligned}
\frac{d S(t)}{d t}= & 5+0.6 R(t)+0.3 Q(t)-0.0002 S(t) E(t) \\
& -0.0003 S(t) I(t)-0.0001 S(t), \\
\frac{d E(t)}{d t}= & 0.0002 S(t) E(t)+0.0003 S(t) I(t) \\
& -0.0102 E(t)-0.2 E(t-\tau) \\
\frac{d I(t)}{d t}= & 0.01 E(t)-0.40003 I(t)-0.48 I(t-\tau), \\
\frac{d Q(t)}{d t}= & 0.4 I(t)-0.30001 Q(t)-0.35 Q(t-\tau), \\
\frac{d R(t)}{d t}= & 0.2 E(t-\tau)+0.48 I(t-\tau)+0.35 Q(t-\tau) \\
& -0.60001 R(t) .
\end{aligned}
$$



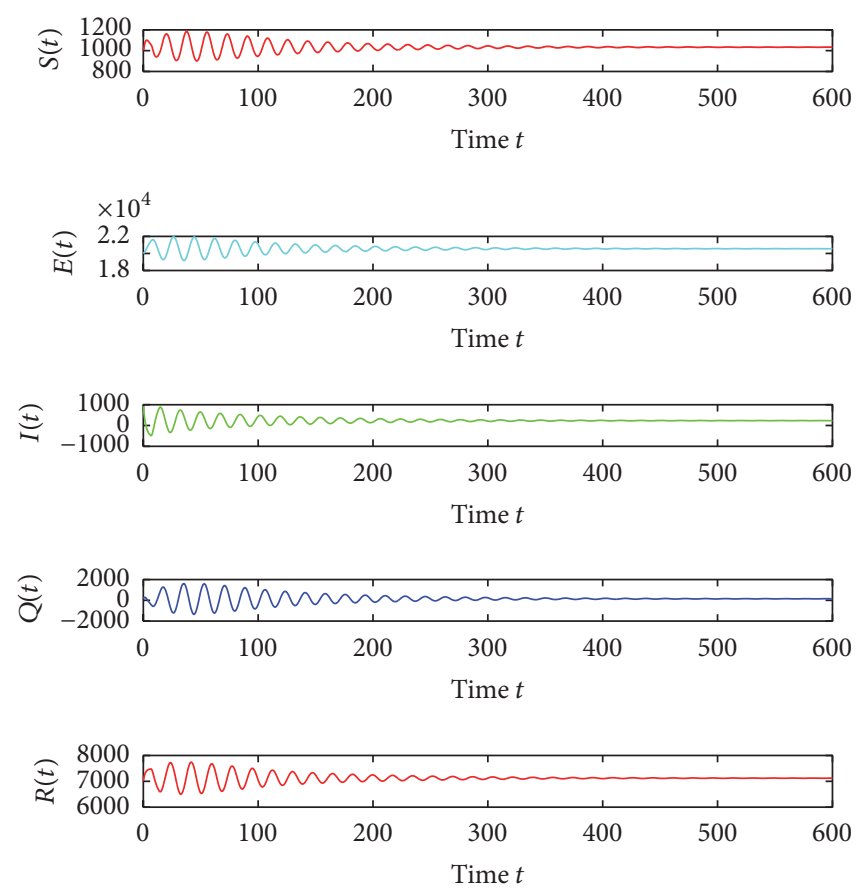

FIGURE 1: $D_{*}$ is locally asymptotically stable when $\tau=6.76<\tau_{0}=7.5508$.

Then, we get $B_{1}=88.0300, B_{2}=0.6153, B_{3}=30.4972$, $B_{4}=0.0210$, and the unique endemic equilibrium $D_{*}(1033.4$, 20526.3334, 233.1743, 143.4698, 7111.1633). Further we have $\operatorname{det}_{1}=A_{4}=9.3093>0, \operatorname{det}_{2}=3.6022>0, \operatorname{det}_{3}=0.4090>$ 0 , det $_{4}=0.2727>0$, and $\operatorname{det}_{5}=0.0154>0$. Thus, $D_{*}$ is asymptotically stable when $\tau=0$.

For $\tau>0$, we obtain $\omega_{0}=1.2030, \tau_{0}=7.5508$, and $\lambda^{\prime}\left(\tau_{0}\right)=0.9208-0.2975 i$ by some complicated computations using Matlab software package. Based on Theorem 1, we know that the endemic equilibrium $D_{*}$ is asymptotically stable when $\tau \in[0,7.5508)$. In this case, propagation of the worms can be controlled easily and this is exhibited by numerical simulation shown in Figure 1. However, once the magnitude of the time delay $\tau$ passes through the critical value $\tau_{0}=$ $7.5508, D_{*}$ will lose its stability and a Hopf bifurcation occurs. In this case, the worm propagation will become unstable and propagation of the worms will be out of control, which can be illustrated in Figure 2. The bifurcation phenomenon can be also exhibited by the bifurcation diagram shown in Figure 3. It follows from $(46)$ that $C_{1}(0)=-0.9606-0.2731 i, \mu_{2}=$ $1.0432>0, \beta_{2}=-1.9212<0$, and $T_{2}=0.0642$. Thus, according to Theorem 2, the bifurcation at $\tau_{0}=7.5508$ is supercritical and stable, and the period of the Hopf bifurcation increases.

\section{Conclusions and Further Developments}

A delayed SEIQRS model with graded infection rates for Internet worms is proposed in this paper based on the model in the literature [18]. Compared with the models investigated in the previous literatures, the exposed and the infectious computers in the model considered in our paper have different infection rates. Also, we incorporate the time delay due to the period that the antivirus software uses to clean the worms in the exposed, the infectious, and the quarantined computers. Therefore, the model considered in our paper is more general.

We mainly investigate the effect of the time delay $\tau$ on the stability of the model. Based on the numerical simulations presented in our paper, we found that when the value of the delay $\tau$ is below the critical value $\tau_{0}$, the model is locally asymptotically stable, which means that the number of the five classes computers in system (2) will be in an ideal steady state and we can control prevalence of the worms in (2) easily. However, once the value of the delay $\tau$ is above $\tau_{0}$, a Hopf bifurcation occurs. This phenomenon suggests that the numbers of computers of the five classes in system (2) will fluctuate periodically in a range. This is not helpful to control the prevalence of the worms. Hence, we should control the phenomenon by combining some bifurcation control strategies and other relative features of virus prevalence, for example, topological structures of networks, which is our future work.

Thus, our further research directions include the possibility of linking the results obtained with the model proposed in the present paper with the results coming from the networks theory. Specifically, the interest focuses on the possibility of gaining a deep understanding of the impact of the network topology on the viral prevalence.

\section{Conflicts of Interest}

The authors declare that they have no conflicts of interest. 

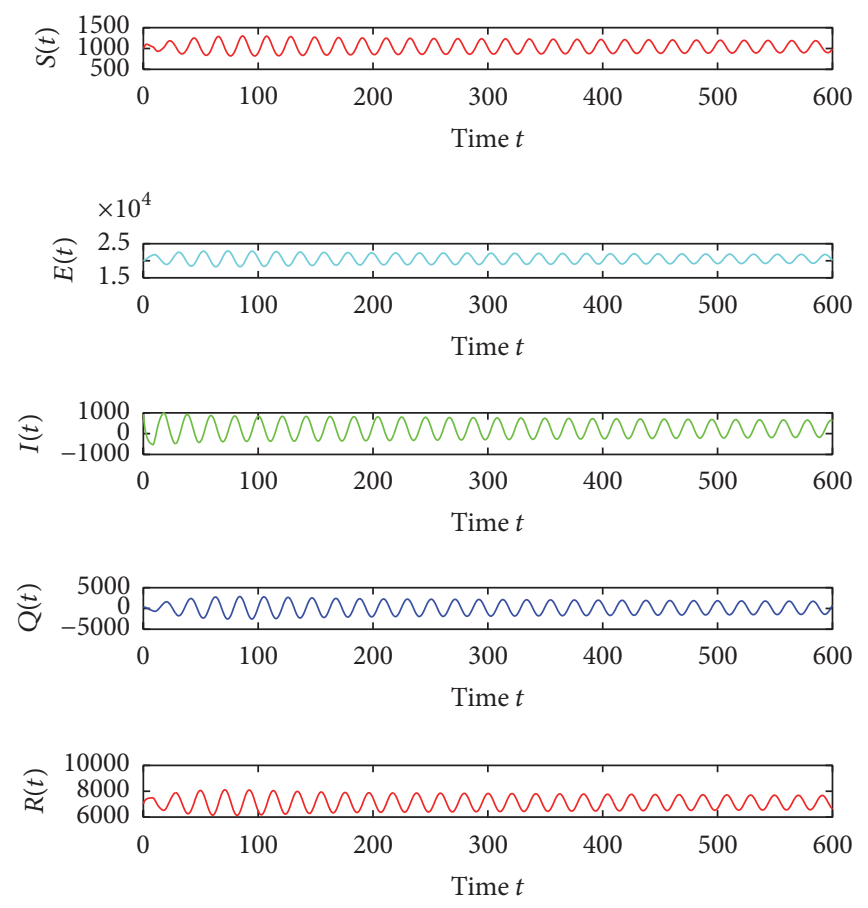

FIgURE 2: $D_{*}$ loses its stability when $\tau=8.42>\tau_{0}=7.5508$.

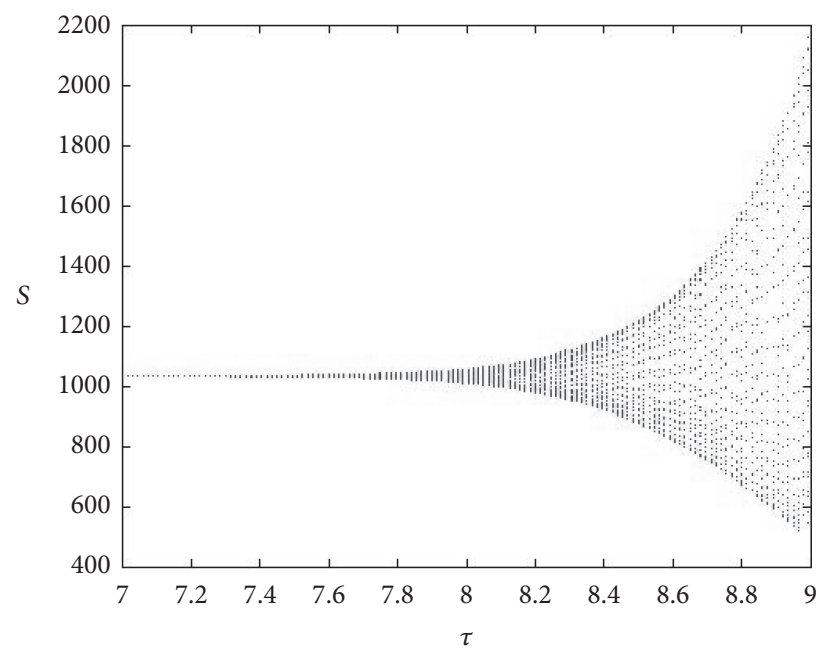

FIgURE 3: Bifurcation diagram with respect to $\tau$.

\section{Acknowledgments}

This research was supported by Natural Science Foundation of Anhui Province (no. 1608085QF145) and Natural Science Foundation of the Higher Education Institutions of Anhui Province (no. KJ2014A006).

\section{References}

[1] L. A. Goldberg, P. W. Goldberg, C. A. Phillips, and G. B. Sorkin, "Constructing computer virus phylogenies," Journal of Algorithms, vol. 26, no. 1, pp. 188-208, 1998.
[2] L. A. Gordon, M. P. Loeb, W. Lucyshyn, and R. Richardson, "CSI/FBI Crime and Security Survey," Information Management and Computer Security, vol. 15, pp. 78-101, 2000.

[3] T. Dong, A. Wang, and X. Liao, "Impact of discontinuous antivirus strategy in a computer virus model with the point to group," Applied Mathematical Modelling, vol. 40, no. 4, pp. 3400-3409, 2016.

[4] N. Sharma and A. K. Gupta, "Impact of time delay on the dynamics of SEIR epidemic model using cellular automata," Physica A: Statistical Mechanics and its Applications, vol. 471, pp. 114-125, 2017. 
[5] F. Wang, Y. Zhang, C. Wang, J. Ma, and S. Moon, "Stability analysis of a SEIQV epidemic model for rapid spreading worms," Computers \& Security, vol. 29, no. 4, pp. 410-418, 2010.

[6] B. K. Mishra and N. Keshri, "Mathematical model on the transmission of worms in wireless sensor network," Applied Mathematical Modelling, vol. 37, no. 6, pp. 4103-4111, 2013.

[7] B. K. Mishra and D. K. Saini, "SEIRS epidemic model with delay for transmission of malicious objects in computer network," Applied Mathematics and Computation, vol. 188, no. 2, pp. 14761482, 2007.

[8] X. Xiao, P. Fu, C. Dou, Q. Li, G. Hu, and S. Xia, "Design and analysis of SEIQR worm propagation model in mobile internet," Communications in Nonlinear Science and Numerical Simulation, vol. 43, pp. 341-350, 2017.

[9] T. Dong, X. Liao, and H. Li, "Stability and Hopf bifurcation in a computer virus model with multistate antivirus," Abstract and Applied Analysis, vol. 2012, Article ID 841987, 2012.

[10] M. Peng, X. He, J. Huang, and T. Dong, "Modeling computer virus and its dynamics," Mathematical Problems in Engineering, vol. 2013, Article ID 842614, 5 pages, 2013.

[11] R. K. Upadhyay, S. Kumari, and A. K. Misra, "Modeling the virus dynamics in computer network with SVEIR model and nonlinear incident rate," Applied Mathematics and Computation, vol. 54, no. 1-2, pp. 485-509, 2017.

[12] B. K. Mishra and N. Jha, "SEIQRS model for the transmission of malicious objects in computer network," Applied Mathematical Modelling, vol. 34, no. 3, pp. 710-715, 2010.

[13] X. Yang, B. Liu, and C. Gan, "Global stability of an epidemic model of computer virus," Abstract and Applied Analysis, vol. 2014, Article ID 456320, 2014.

[14] L.-X. Yang, X. Yang, L. Wen, and J. Liu, "A novel computer virus propagation model and its dynamics," International Journal of Computer Mathematics, vol. 89, no. 17, pp. 2307-2314, 2012.

[15] L.-X. Yang, X. Yang, Q. Zhu, and L. Wen, "A computer virus model with graded cure rates," Nonlinear Analysis: Real World Applications, vol. 14, no. 1, pp. 414-422, 2013.

[16] M. Yang, Z. Zhang, Q. Li, and G. Zhang, "An SLBRS model with vertical transmission of computer virus over the internet," Discrete Dynamics in Nature and Society, vol. 2012, Article ID 925648, 2012.

[17] F. Wang, Y. Yang, D. Zhao, and Y. Zhang, "A worm defending model with partial immunization and its stability analysis," Journal of Communications, vol. 10, no. 4, pp. 276-283, 2015.

[18] F. Wang, F. Yang, Y. Zhang, and J. Ma, "Stability analysis of a seiqrs model with graded infection rates for internet worms," Journal of Computers, vol. 9, no. 10, pp. 2420-2427, 2014.

[19] L. Feng, X. Liao, H. Li, and Q. Han, "Hopf bifurcation analysis of a delayed viral infection model in computer networks," Mathematical and Computer Modelling, vol. 56, no. 7-8, pp. 167-179, 2012.

[20] Z. Zhang and D. Bi, "Dynamical analysis of a computer virus propagation model with delay and infectivity in latent period," Discrete Dynamics in Nature and Society, vol. 2016, Article ID 3067872, 2016.

[21] Z. Zhang and H. Yang, "Hopf bifurcation of an SIQR computer virus model with time delay," Discrete Dynamics in Nature and Society, vol. 2015, Article ID 101874, 8 pages, 2015.

[22] J. Liu, C. Bianca, and L. Guerrini, "Dynamical analysis of a computer virus model with delays," Discrete Dynamics in Nature and Society, vol. 2016, Article ID 5649584, 21 pages, 2016.
[23] Z. Zhang and D. Bi, "Bifurcation analysis in a delayed computer virus model with the effect of external computers," Advances in Difference Equations, vol. 317, pp. 1-13, 2015.

[24] Z. Zhang, Y. Wang, and M. Ferrara, "Stability and Hopf bifurcation for a delayed computer virus model with antidote in vulnerable system," Journal of Control Science and Engineering, vol. 2017, Article ID 9360430, 9 pages, 2017.

[25] E. Javidmanesh, Z. Afsharnezhad, and S. Effati, "Existence and stability analysis of bifurcating periodic solutions in a delayed five-neuron BAM neural network model," Nonlinear Dynamics, vol. 72, no. 1-2, pp. 149-164, 2013.

[26] C. J. Xu, X. H. Tang, and M. X. Liao, "Stability and bifurcation analysis of a six-neuron BAM neural network model with discrete delays," Neurocomputing, vol. 74, no. 5, pp. 689-707, 2011.

[27] B. D. Hassard, N. D. Kazarinoff, and Y.-H. Wan, Theory and Applications of Hopf Bifurcation, Cambridge University Press, 1981.

[28] X.-Y. Meng, H.-F. Huo, X.-B. Zhang, and H. Xiang, "Stability and Hopf bifurcation in a three-species system with feedback delays," Nonlinear Dynamics, vol. 64, no. 4, pp. 349-364, 2011.

[29] R. K. Upadhyay and R. Agrawal, "Dynamics and responses of a predator-prey system with competitive interference and time delay," Nonlinear Dynamics, 2015.

[30] C. Bianca, M. Ferrara, and L. Guerrini, "The Cai model with time delay: existence of periodic solutions and asymptotic analysis," Applied Mathematics \& Information Sciences, vol. 7, no. 1, pp. 21-27, 2013. 


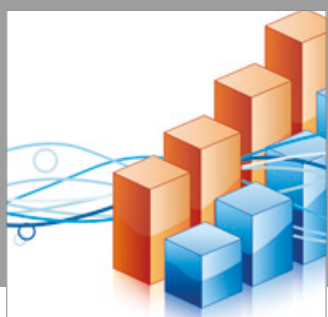

Advances in

Operations Research

vatersals

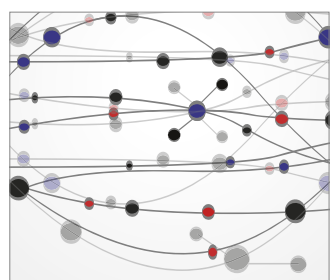

\section{The Scientific} World Journal
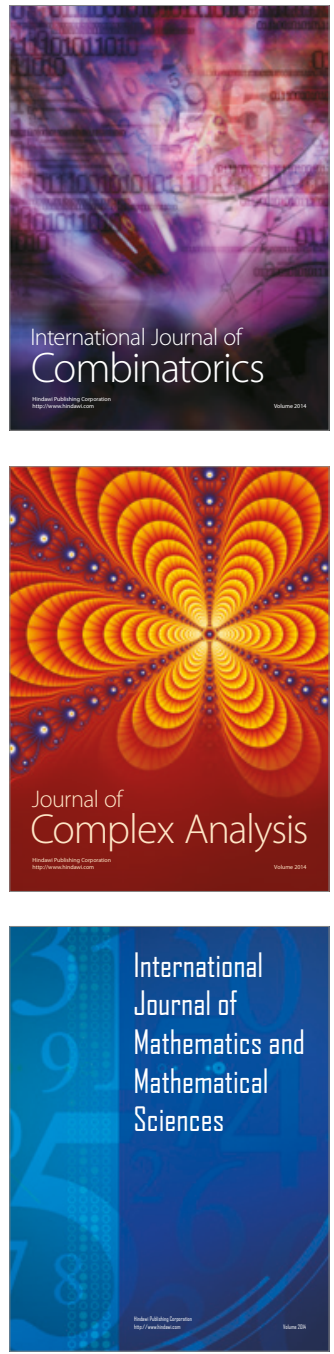
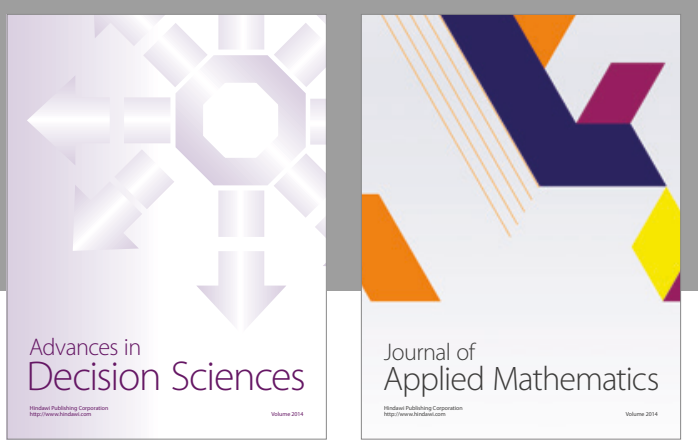

Algebra

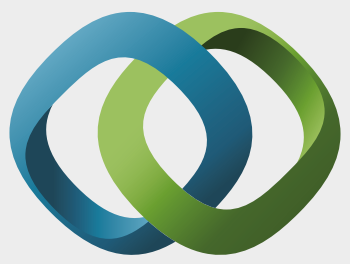

\section{Hindawi}

Submit your manuscripts at

https://www.hindawi.com
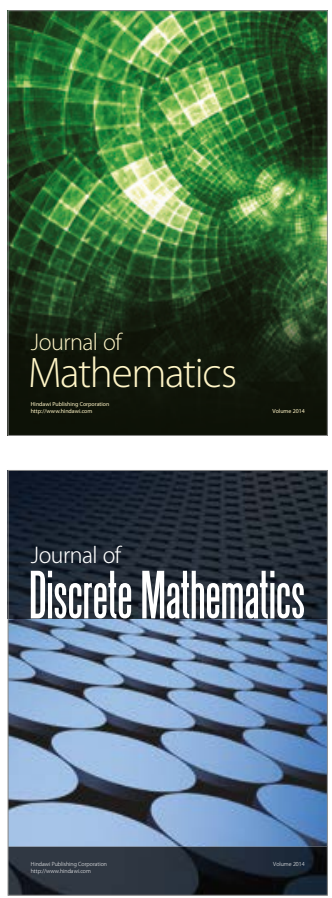

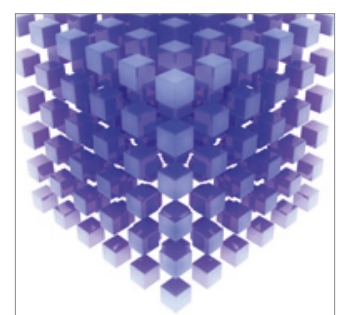

Mathematical Problems in Engineering
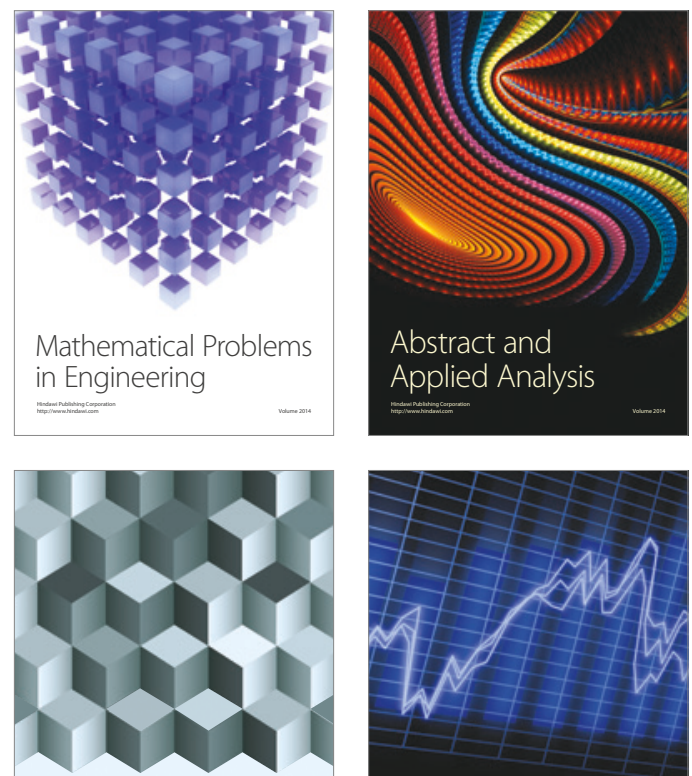

Journal of

Function Spaces

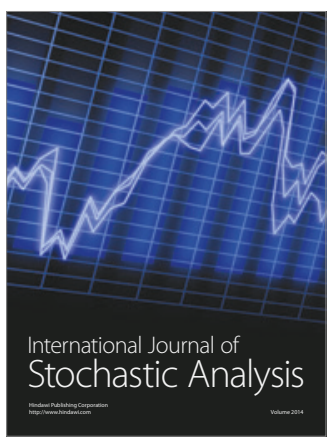

Probability and Statistics
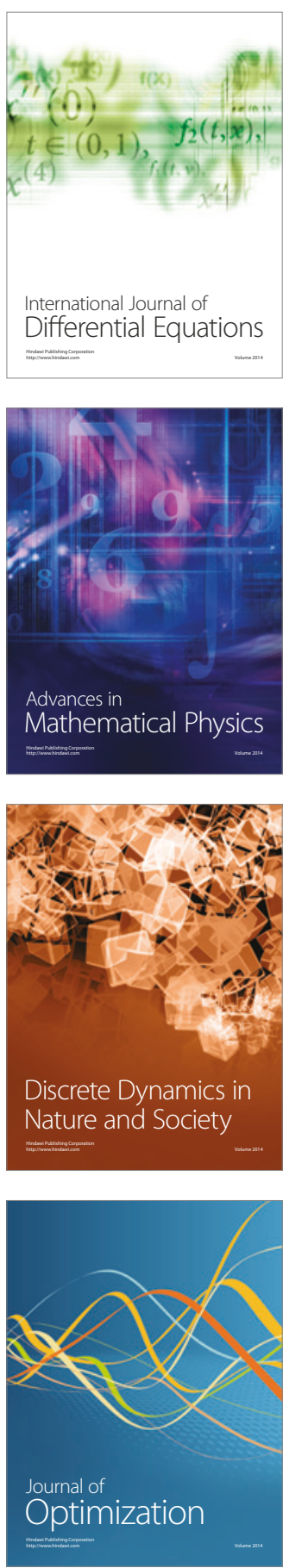